\section{FOREIGN BODIES IN THE AIR AND FOOD PASSAGES.}

\section{REMARKS ON FIVE CASES.} BY

C. A. SCOTT RIDOUT, M.S.Lond., F.R.C.S.Eng., SURGEON TO THE EAR, NOSE, AND TAROAT DEPARTMENT OF THE PORTSMOUTH AND SOUTHERN COUNTIES EYE AND EAR HOSPITAL: ASSISTANT SURGEON TO THE ROYAL PORTSMOUTH GOSPITAL.

The following series of 5 cases of foreign bodies in the air and food passages form an inleresting group illustrative of the value of modern treatment for these conditions, and of the necessity of having at hand the most up-to-date instruments for dealing with them; this was shown especially by the one failure to remove the foreign body by endoscopic methods, recourse to the older and cruder surgical method of external incision becoming a necessity.

CASE I.-Toothplate Impacted in the Right Bronchus.

J. S., a mechanic aged 22, was referred to the Eye and Ear Hospital by $\mathrm{my}$ colleague Mr. Bosworth Wright on October $25 \mathrm{th}$, 1922, with the following history. On October $24 t$ he lay down about 6 p.m. and fell asleep; he woke ab.ut an hour later, coughing, and felt pain in the right side of the chest. He then missed his lower toothplate, which contained ten teeth ard had one holding hook. Past history elicited that on a few occasions he had suffered from petit mal, becoming unconscious for a short period. The cough and pain in the chest continued, and he went to the Royal Portsmouth Hospital, where by screening with $x$ rays a foreign body was located to the right of the middle line of the chest opposite the fifth rib posteriorly. An oesopbageal bougie passed easily. He was transferred to my care next day, and on

further screening the foreign body was seen in the same place as before to the right of the middle line of the chest, whether screeued from front or back. A radiograph confirmed the appearance seen in the screen. Cough was frequent and painful. Loud rhonchi were heard over the centre of the right pulmonary area. It was obvious therefore that a foreign body was present in the right broncbus, but its s.ze (length as seen about 11 inches) did not correspond to the description of the missing tootnplaie, though its contour corresponded to a smaller plate than the one described.

The patient was then anaesthetized by Dr. C. Mayhew, anaesthetist to the Eye and Ear Hospital, with a C.E. mixture, after injection of the pre-tracheal tissues with 2 per cent. novocain and adrenaline 1 in 20,000 . A deliberate tracheotomy was performed, the second, third, fourth, and fifth tracheal rings being incised after division of the isthmus of the thyroid and injection of 1 per cent. cocaine into the trachea. The interior of the traclea was then mopped with 5 per cent. cocaine-as far as the bifucation, coughing being thereby prevented. The ancesthetic was now changed to oxygen and chloroform, given through a Eustachian calheter directly into the trachea.

Irwin Moore's internal illuminating bronchoscope was then introduced into the right brouchus, where the curve of the hook attached to the vulcanite body of the toothplate was seen. By the aid of I'win plate was seen. By the aid of I'win brought to the tracheotomy wound, where, unfortunately, it slipped from the forceps grip, aud, before the assistant was able to secure it, was sucked back into the trachea and again lodged in the right bronchus. It was again seen, seized, and brought upwards, but once more slipped from the forceps grip (the vulcanite surface was rendered extremely difficult to ho.d owing to much mucus) while still in the trachea and this time fell back into the left bronchus, where it was ultimately secured and bruaght out through the tracheotomy wound. The trachea and bronchi had

wound. The trachea and bronchi had previously to this been mopped with 20 per cent. cocaine as the
frequent manipulations had rendered their surface irritable.

The plate when removed was found to contain the hook end a portion of which was missing, and five tooth sockets holding three teeth, two being empty, and the plate had evidently been broken exactly in the middle line. In order to be quite sure that nothing was left behind the bronchial tree was explored on both sides with the bronchoscope, a good view being obtained. The patient subsequently pussed the other half of the plate with five teeth in five sockets per rectum, having evidently smashed the plate in half while in an epileptic spasm, and broken off two teeth as well-these latter were not recovered. After-progress was uneventful save for some mild bronchitis, for whioh due precautions were taken.
Noteworthy points about the case are:

(a) It would seem that, in similar cases where large and slippery foreign bodies are present in the bronchi, the position of the patient should be oblique with head slanting downwards in case of slipping of the forceps grip.

(b) The assistant should be ready with a pair of Spencer Wells forceps to secure a good grip on the foreign body whenever it appears in the wound.

(c) 'Tracheotomy would seem advisable in this class of case, as the foreign body was too large to be drawn through the bronchoscope, and had-to be brought up together with the withdrawal of the tube, and would otherwise have certainly damaged the glottis.

(d) Anaesthesia as carried out in this case, by means of chloroform and oxygen administered through a large-size Eustachian catbeter introduced alongside the bronchoscope, proved very satisfactory.

CASE II.-Fortign Body in the Hypopharynx just Above the Entrance to the Verophanus.

M. K., a schoolgirl 9 years of age, was admitted to the Portsmouth Eye and Ear Hospital on September 18th, 1922, with a history of having swallowed a drawing-pin while at school. A radiograph showed the drawing-pin lying slightly to the right of the middle line opposite the ring of the cricoid cartilage. The pitient was anaesthetized with C.E. mixture and an oesophagoscope introduced. At first the foreign body was not seen, the oesophlgus being entered and found normal in appearance; but on withdrawing and examining the right lateral wall of the hypopharynx it was easily observed and removed with forceps.

The value of endoscopic methods was well illustrated in this case as any blind manipulation with coin catchers, etc., could only have been productive of injury owing to the sharp point of the drawing-pin, and
the danger of pushing it downwards with a probang or other means is obvious. External incision wou'd have necessitated a severe operation and probably a tedious convalescence.

CASE III.-Mass of Fish Bones Impacted Across the Oesophagus Opposite the Sterno clavicular Articulation.

Mrs. A. J. was seen by me in consultation with Dr. Lyth of Southsea in the spring of 1921 , with a history of having swailowed a mass of fish bones whilst eating a meal, and that this was followed by great pain radiating over the lower neck and clavicular region on both sides, and inability to swallow.

The patient was anaesthe.ized and the oesophagoscope introduced, when the fish bone mass was seen lying transversely across the oesophagus opposite the site of pain-that is, the interwith forceps and easily came away, affording complete relief.

This case, again, shows the advantages of endoscopic methods as opposed to the more baıbarous probang which in olden days alone would liave been available; a mass of bone thus situated could easily wound the walls of the oesophagus, and in such a position suppuration or damage to large vessels in the vic nity might easily prove fatal.

C A S E I v.-Toothilate Impacted in the Oesophagus; Failurs to litmoce it oving to Lack of Necessa, y Ap;liances.

A. B., a female patient aged about 35 , was admitted t 5 the Royal Ports nouth Hospital in Septes,ber, 192l, with a history of having swallowed a metal toothplate with pointed lateral extremities but no hocks. A radiograph confirmed the history, and showed the plate lying horizoita!ly across the oesophagus just be.ow the riug of the cricoid.

Under local anaesthesia an oesophagoscope was introduced and the upper edge of the plate easily located; the plate was seized with forceps, but could not be shifted owing to the fixity of its pointed lateral extremities in the walls of the oesophagus.

As I was not then in possession of Irwiv Moore's shears for dividing such plates in the middle line and removing them in two halves, freeing their points of attachment in this way, and also not then having Paterson's recent expanding oesophac'cscope, I was unable to continue endoscopic methods and had to have recourse of necessity to external oesophagotomy. 'This I performe.l through a somewhat unusual route. As I knew exactly the location of the toothplate, I incised the middle line of the neck. divided the isthmus of the thyroid, and retracted the left lobe of the thyroid away from the trachea and opened the antero-lateral 
aspect of the oesophagus. Having removed the foreign body, I closed the oesophageal wound and allowed the left lobe of the thyroid to fall back, placing a small drainage tube down to the oesophageal incision, and otherwise closed the skin wound. A temporary oesophageal fistula supervened, but healed within a fortnight; otherwise after-progress was uneventful.

The failure to remove the foreign body in this case by endoscopic methods was entirely due to lack of the necessary implements. External oesophagostomy in the face of modern appliances must now be regarded as unnecessary, if not almost bad surgery, when applied to foreign bodies of a nonpenetrating variety; and when one contrasts the rapid recovery with the minimum of inconvenience to the patient of endoscopic methods, as compared with the more serious character of and prolonged convalescence from external surgical procedures on the oesophagus, the latter should be con. sidered by all surgeons as out of date, unless the circumstances are very ex. ceptional; such methods as blindly groping with probangs, etc., should only be mentioned to be condemned.

CASE v.-Meccano Wheel Impacted in the Oesophagus Opposite the T'hird Dorsal Vertebra.

The patient, a boy aged $6 \frac{1}{2}$, was admitted to the Portsmouth Eye and Ear Hospital on December 5th, 1922, with a history of having swallowed a meccano wheel, through which be was trying to whistle. The radiograph (Fig. 3) shows its position as stated above.

This is the type of case where up to recent years (and even possibly nowa. days) a probang would be used to push the foreign body downwards, to be passed per rectum, with probably no great inconvenience to the patient;

but the ease with which it can be seen and removed by endoscopic methods, as was dono in this case, with the elimination of any possible subsequent complications or damage to the oesopliageal walls, still further renders the use of such instruments as the probang inadvisable when other methods are available. As regards the technique to be observed in endoscopic manœurres such as the foregoing cases entailed, the conclusions arrived at from these and other cases are as follows:

1. Position of the Patient.-If the mancurre is to be carried out under a local anaesthetic, which is only possible in a certain proportion of cases, the patient should sit on a low stool, with the head in the "sword-swallowing" position -that is, extended fully, chin pointing upwards; if under a general anaesthetic, the patient should lie in the dorsal position, head over the end of the table, supported so as to bring the chin as nearly as possible level with the promi. nence of the thyroid cartilage. The patient as a whole should be on an inclined plane, head lowest, so as to obviate the occurrence which happened in Case I, of the foreign body slipping back if escaping from the grip of the forceps.

2. Before operation, in the case of adults a hypodermio injection of 1/150 grain of atropine should be given fifteen minutes before commencing, and in the case of children under 12 years of age 5 to 10 drops of tincture of belladonna, according to the age of the child, by the mouth. This will be found very useful in preventing undue accumulations of mucus. In nerrous adults also $1 / 6$ to $1 / 4$ grain of morphine hypodermically will assist by calming the patient.

3. Choice of Anaesthetic. $-\mathrm{As}$ it is of great importance that the field of vision be unimpeded by collections of mucus, C.E. mixture, followed by pure cliloroform, has been found of greatest service. In the case of adults, whose control is good, a local anaesthetic may be used with advantage; it will, however, take from ten to fifteen minutes to prepare the fauces and pharynx by painting with from 5 to 10 per cent. cocaine before com. plete tolerance is obtained, and a stimulant such as sal volatile should be at hand in case of fainting or collapse. Some distinguished endo. scopists claim to use little or no local a naesthetic and to be able to induce their patients to allow them to pass their instruments. One regrets that one's own experience differs from this.

4. Position of Surgeon.-If the patient is under a local anaesthetic, the surgeon stands while the patient sits on a low stool. If the patient is under a general anaesthetic, the surgeon sits on a low stool-a dressing-box malses an admirable seat.

5. Treatment of Tubes.-The tube should be warmed and smeared with glycerin as a lubricant; a cold tube excites spasm.

6. Lastly-a small but practical point-if the surgeon is wearing glasses himself, these should be well warmed or smeared with Sterilla soap, otherwise he is liable to find his own vision fogged just when he most particularly wishes to see clearly.

\section{“LUNG SPLINTING" IN THE TREATMENT OF PULMONARY TUBERCULOSIS.* BY}

SAMUEL HENRY STEWART, M.D.EDIN., MEDIOAL SOPERINTENDENT, BELFAST MUNICIPAL SANATORIOM, WHITEABBEY.

THrs paper represents an attempt to work from evidence ascertainable by the clinician towards treatment in pulmonary tuberculosis. Practically any organ in the body may be attacked by tubercle; the frequency and course of the pulmonary lesion then raises the question of the significance of this particular condition. Fuller appreciation of Koch's experiments, which demonstrated the beliaviour of immunized cells to tuberculous infection, forces the attitude that pulmonary tubercle is a phase in tuberculosis having defensive properties, and probably supplementing the lymphatic system in guarding against spread to other parts. This defensive element must receive consideration in treatment. The majority of serious therapeutic attempts have at their foundation the production of immunity. This phase of tuberculosis is ill defined and the results would appear to justify experiment in supplementary or other directions.

Talking these facts into consideration I studied, on a com. parative basis, various manifestations of tuberculosis. The following observations would seem to establish rest of the diseased organ as a fundamental in treatment. The dis. cussion of the introduction of rest by lung splinting in the treatment of pulmonary tuberculosis can be dealt with most logically under three headings.

\section{Facts which Point to Immobilization as being a} Rational Procedure in Treatment.

I first studied the deaths incident to the various tuberculous lesions. The following list, ${ }^{1}$ representing the deaths in a large Midland city, is cited as a basis of argument:

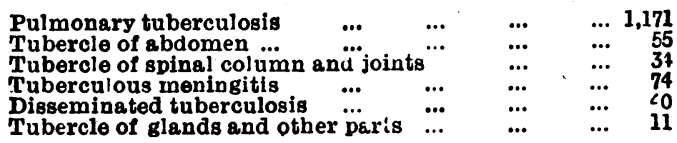

In. the above table we notice that mortality from tuber. culosis is mainly associated with organs whose function involves movement. 'Iubercle in what may be described as stationary organs accounts for a comparatively small number of deatlis.

I next considered this association of tuberculosis and mobile organs clinically. To this end I divided up the various common lesions for comparison as follows: (a) Disease. in the stationary organs-for example, glands; $(b)$ disease in organs which are mobile but capable of being immobilized - for example, joints; (c) disease in mobile organs which are uncontrolled - for example, lungs. The large majority of cases falls into the third category.

Study of these three chronic types of tuberculosis soon 